\title{
Real-world incidence and cost of pneumonitis post-chemoradiotherapy for Stage III non-small-cell lung cancer
}

Kellie J Ryan ${ }^{1}$, Damion Nero*,2, Bruce A Feinberg², Choo Hyung Lee ${ }^{2}$, Rodrigo Pimentel ${ }^{1}$, Ajeet Gajra², Jonathan K Kish² \& Brian Seal ${ }^{1}$

${ }^{1}$ AstraZeneca, US Medical Affairs, Gaithersburg, MD 20878, USA

${ }^{2}$ Cardinal Health Specialty Solutions, Dublin, OH 43017, USA

*Author for correspondence: damion.nero@cardinalhealth.com

\begin{abstract}
Aim: To estimate the real-world incidence and timing of radiation pneumonitis following chemoradiotherapy for Stage III non-small-cell lung cancer and compare costs between patients with and without radiation pneumonitis. Methods: Retrospective analysis using the Symphony Health Integrated Dataverse. Results: Pneumonitis incidence was $12.4 \%$ with a 177 -day mean time to onset. Patients with versus without pneumonitis were more frequently admitted to the hospital (33.8 vs $19.2 \%, p<0.0001$ ) and seen in the emergency room ( 51.9 vs $35.8 \%, p<0.0001)$ and had higher mean total healthcare costs (US $\$ 4251$ vs US $\$ 3969$ per-patient per-month; $p=0.0163$ ). Conclusion: Although pneumonitis significantly increased healthcare resource utilization and costs in chemoradiotherapy-treated stage III non-small-cell lung cancer, the per-patient per-month differential was $<10 \%$. Such financial assessments are critical for cost-benefit analysis.
\end{abstract}

First draft submitted: 28 August 2019; Accepted for publication: 5 November 2019; Published online: 5 December 2019

Keywords: burden of disease $\bullet$ chemoradiotherapy $\bullet$ cost $\bullet$ non-small-cell lung cancer $\bullet$ pneumonitis

Chemoradiotherapy (CRT) is the standard of care for patients with unresectable stage III non-small-cell lung cancer (Stage III NSCLC) [1]. The annual incidence rate of NSCLC was reported as 38.61 per 100,000 patients in 2016 [2]. CRT has been shown to significantly improve overall survival in unresectable, Stage III NSCLC patients across a number of randomized clinical trials [3-5]. While CRT provides a substantial clinical and survival benefit, it is also associated with toxicity. Radiation induced lung injury (RILI) is an adverse event associated with CRT. RILI can manifest as radiation pneumonitis for up to a year after completion of CRT or as radiation fibrosis beyond that period and at high severity grades is associated with significant morbidity and mortality $[6,7]$. Radiation-related pneumonitis can occur as early as 4 weeks but in some cases 12 months post treatment [8-10].

Durvalumab, a PD-L1 inhibitor, has become the new standard of care as consolidation therapy after CRT for unresectable Stage III NSCLC patients, having conferred improvements in the 12 and 18 month progression-free survival rates (55.9/44.2\% with durvalumab vs $35.5 / 27.0 \%$ with placebo respectively) in the Phase III PACIFIC trial. In the PACIFIC trial, the incidence of all-grade pneumonitis in patients with an adverse event was $12.6 \%$ in the durvalumab arm versus $7.7 \%$ in the placebo arm and grade $3 / 4$ at 1.9 versus $1.7 \%$ respectively [11].

Based on our review of the literature no published studies have described the real-world incidence of pneumonitis in Stage III NSCLC patients receiving CRT [12]. Here, we describe the results of a claims-based analysis, for which the primary objectives were to estimate the real-world incidence and timing of pneumonitis post-CRT initiation for Stage III NSCLC and to compare treatment costs between patients with and without pneumonitis.

\section{Materials \& methods}

\section{Data source}

This was a retrospective analysis using the linked longitudinal claims database Symphony Health Integrated Dataverse (IDV), conducted in accordance with International Society for Pharmacoeconomics and Outcomes

Future $\because \cdots$ Medicine 


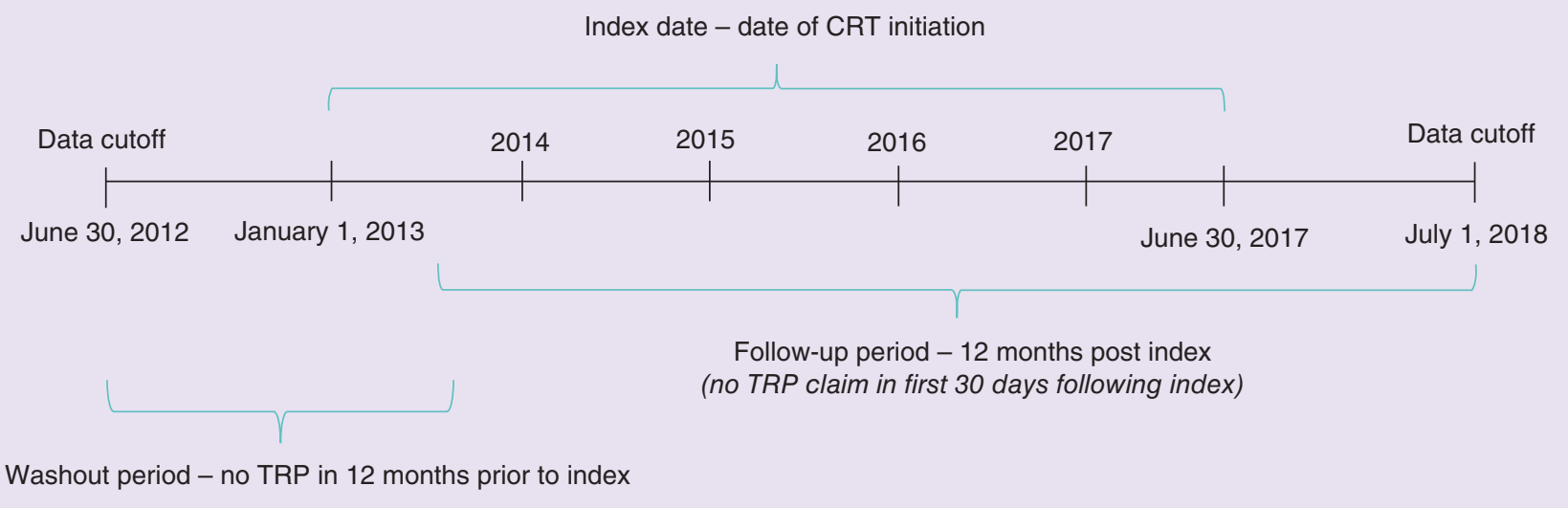

Figure 1. Study design: definition of incident population. CRT: Chemoradiation; TRP: Treatment-related pneumonitis.

Research guidelines for retrospective studies [13]. The Symphony Health IDV integrates data from physician practices, pharmacies and hospitals, providing a broad longitudinal view of healthcare delivery and patient usage patterns that are representative of the US population across age, sex, geography and payment type (including commercial, Medicare and Medicaid plans). Medical, pharmacy and hospital claims data are linked through a common de-identified patient field with a unique code for each patient. The data source contains claims for 280 million active unique patients, representing over $73 \%$ of specialty prescriptions, $58 \%$ of medical claims and $30 \%$ of hospital claims volume in the US. Currently, these data are collected from approximately 903,500 sources, covering 13.1 million employer groups and 1.8 million prescribers.

\section{Patient identification}

Patients with Stage III NSCLC who received CRT between 01/01/2013-06/30/2017 (study period) were selected for this analysis (Figure 1). Patients were followed from the initiation of CRT therapy with the index date defined as the date of the initiation of the chemotherapy regimen or radiation therapy (whichever came first) used for CRT. The inclusion criteria included a diagnosis of lung cancer (ICD-9 codes 162.2-162.5, 162.8, 162.9; ICD-10 codes C34.00-C34.02, C34.10-C34.12, C34.2, C34.30-C34.32, C34.80-C34.82, C34.90-C34.92) during the study period, no claims for surgical resection related to lung cancer, and receipt of treatment with CRT per NCCN guidelines for NSCLC [1]. Patients who had $\geq 5$ radiation claims within 45 days of chemotherapy start were defined as receiving concurrent CRT; those who had $\geq 5$ radiation claims 45 days after chemotherapy start were defined as receiving sequential CRT. Patients were also required to be age $\geq 18$ years at their earliest claim for CRT, to have at least 12 months of claims activity prior to CRT treatment (baseline period), and to have at least 12 months of claims activity post index date. Claims activity during a quarter was used in lieu of enrollment data that are not available in the IDV. The exclusion criteria included participation in a clinical trial at any time after lung cancer diagnosis (ICD-10 code Z00.6; ICD-9 code V70.7; HCPCS codes G0276, G0293, G0294, S9988, S9990, S9991, G9057, S9992, S9994, S9996; or Modifier Codes Q0, Q1), diagnosis of small cell lung cancer (defined as patients who received irinotecan, temozolomide or topotecan following CRT and patients who received prophylactic cranial irradiation (CPT code 77470) any time during the study period), any history of pneumonitis prior to CRT and diagnosis or treatment of a second primary malignancy prior to CRT. To limit the patient population to Stage III NSCLC patients, patients with secondary malignancy codes (ICD-9 codes 196.XX, 197.XX, 198.XX or ICD-10 codes C77.XX, C78.XX, C79.XX) during their initial CRT were also excluded. Baseline demographic and clinical characteristics, including comorbidities, were assessed during the baseline period.

Incident pneumonitis was defined using a set of ICD-9/ICD-10 codes indicating a diagnosis of pneumonitis (ICD-9 codes 495.9, 516.30, 516.32, 516.33, 516.35, 516.36, 516.39; ICD-10 codes J67.9, J84.11X); pulmonary manifestations due to radiation or other sources (ICD-9 codes: 508.0, 508.1, 508.8, 508.9, ICD-10 codes J70.0, $\mathrm{J} 70.1, \mathrm{~J} 70.2, \mathrm{~J} 70.3, \mathrm{~J} 70.4, \mathrm{~J} 70$ ) or pulmonary conditions (ICD-9 code 518.89). While pneumonitis diagnosis codes are present within the ICD code list, in a clinical setting pneumonitis can be a diagnosis based on exclusion of other 
pulmonary conditions that appear in Stage III NSCLC patients and, therefore, challenging to define this diagnosis in administrative claims data. Given this, other codes such as those for pulmonary disorders or manifestations due to radiation treatment are used in addition to pneumonitis specific codes to capture potential pneumonitis in claims data [14,15]. Patients were identified as having an incident case if they had no history of pneumonia/pneumonitis prior to CRT treatment and excluding pneumonia/pneumonitis diagnosis within 30 days after CRT treatment to control for post-obstructive pneumonia. The cumulative incidence of pneumonitis was reported as the proportion of CRT patients with incident pneumonitis within 12 months of CRT treatment while the incidence rate was reported as the number of pneumonitis cases per 1000-person months of follow-up post CRT.

\section{Assessment of pneumonitis management \& healthcare resource utilization}

Medical management of pneumonitis was assessed for patients with a diagnosis of pneumonitis based on the above criteria. Medical management included patients receiving therapy for treatment of pneumonitis (e.g., treatment with intravenous [IV] steroids such as methylprednisolone and dexamethasone) or patients being admitted to a setting where their pneumonitis would be monitored (e.g., an ER or inpatient admission). The frequency of medical management approach for patients with Stage III NSCLC was reported during the follow-up period.

Healthcare resource utilization was evaluated using admission codes for hospitalization, ER and office visits and other outpatient visits. Setting of care were defined using the Centers for Medicare \& Medicaid Services (CMS) place of service (POS) codes or claim type codes in the IDV. Settings of care included inpatient, office visits, emergency room (ER) and other outpatient visits (outpatient). Direct medical costs were calculated based on standardized costs. All standardized drug costs were based on CMS 2017 average sales price (ASP) [16] for infused and injectable drugs and average wholesale price (AWP) from First Data Bank for oral drugs [17]; standardized procedure costs were based on Medicare Physician Fee Schedule (MPFS) [18], Hospital Outpatient Payment System (OPPS) and Clinical Laboratory Fee Schedule (CLAB) from CMS [19].

For inpatient costs, the HCUP [20] cost per hospitalization day were used to account for room and board, procedures and medications that are not itemized on the claim. For drugs and procedures that were itemized on the claim, we used MPFS facility standardized costs for procedures and CMS ASP and WAC costs for drugs. Costs and utilization were reported per-patient per-month (PPPM). All costs were adjusted to 2018 dollars.

\section{Analysis}

Descriptive analyses assessing patient baseline and clinical characteristics, and economic outcomes (resource utilization and costs) were conducted using means, standard deviations (SDs), and medians for continuous variables and frequencies and proportions for categorical variables. Comparisons between patients with and without incident pneumonitis were performed using t-tests for continuous variables and chi square tests for categorical variables. Nonparametric equivalents to these tests (including the Mann-Whitney U test and Fisher Exact Test respectively) were used in cases were normality of results could not be assumed. All analysis was performed using SAS 9.4.

\section{Results}

\section{Study population}

We identified 7559 Stage III NSCLC patients treated with CRT in the Symphony Health IDV, of which 5979 patients did not have pneumonitis in the baseline period. Of those, 742 patients had incident pneumonitis identified during the 12 months post CRT initiation. The majority of the population of patients with incident pneumonitis (739 patients) had concurrent CRT while only three patients had sequential CRT. The cumulative incidence of treatment-related pneumonitis was $12.4 \%(742 / 5,979)$ with an annual incidence rate ranging from 5.5 to $18.1 \%$. The average incidence rate (cases/1000-person months) across the study period was 126.4 (95\% CI: 117.4-135.8). Mean time to incident pneumonitis was 177 days (median, 169 days).

Demographic and clinical characteristics are presented in Table 1. Of the 5979 NSCLC patients treated with CRT that were identified, the mean age at the time of treatment was approximately 66.9, the population was evenly distributed based on gender (50.7\% female vs $49.3 \%$ male) and the mean Quan-adapted Charlson comorbidity index (CCI) score was 5.2. Overall, no significant differences were observed in the demographic characteristics between patients with and without pneumonitis. Regarding clinical characteristics, patients with pneumonitis had a longer median follow-up from the index date (22.5 vs 20.45 months) and a higher baseline CCI at CRT initiation $(5.85$ vs $5.13, \mathrm{p}<0.0001)$. A significantly higher proportion of patients with pneumonitis had chest pain, dyspnea, fatigue and interstitial lung disease at baseline ( $\mathrm{p}<0.001$, see Table 2 for details). While the mean length 
Research Article Ryan, Nero, Feinberg et al.

Table 1. Demographic and clinical characteristics.

\section{Pneumonitis $\mathrm{n}=\mathbf{7 4 2}$}

Gender (n, \%)

$\begin{array}{ll}\text { Male } & 374 \\ \text { Female } & 368\end{array}$

Age at earliest claim for NSCLC (years)

Mean (SD) 66.85

Median (min, max) 68

$(33.0,79.0)$

$50.4 \%$

$49.6 \%$

2665

$49.1 \%$

$50.9 \%$

No pneumonitis $\mathrm{n}=\mathbf{5 2 3 7}$

p-value

Age at earliest claim for CRT (years)

Mean (SD) 67.01

Median (min, max)

68

(8.1)

$(33.0,79.0)$

66.72

(8.2)

$(36.0,79.0)$

68

(8.2)

$(36.0,79.0)$

US region (n, \%)

Northeast

149

$20.1 \%$

$26.0 \%$

South

193

162

232

West

232

Unknown

6

Primary payer (n, \%)

Assistance programs

$16 \quad 2.2 \%$

Cash 11

Commercial

300

Managed Medicaid

Medicaid

300

38

25

352

0

$21.8 \%$

$31.3 \%$

$0.8 \%$

$2.2 \%$

$1.5 \%$

$40.4 \%$

$5.1 \%$

$3.4 \%$

$47.4 \%$

$0.0 \%$

Unknown

66.89

68

$(36.0,79.0)$

Year of diagnosis of lung cancer $(n, \%)$

\begin{tabular}{|lllll}
\hline 2013 & 154 & $20.8 \%$ & 528 & $10.1 \%$ \\
\hline 2014 & 230 & $31.0 \%$ & 1034 & $19.7 \%$ \\
\hline 2015 & 135 & $18.2 \%$ & 1149 & $21.9 \%$ \\
\hline 2016 & 156 & $21.0 \%$ & 1769 & $33.8 \%$ \\
\hline 2017 & 67 & $9.0 \%$ & 757 & $14.5 \%$ \\
\hline
\end{tabular}

Quan-adapted Charlson comorbidity index (CCl)

Mean (SD) $\quad 5.85$

Median (min, max) 6.0

Baseline comorbidities, (n, \%)

\begin{tabular}{|c|c|c|c|c|c|}
\hline Arrhythmia & 12 & $1.6 \%$ & 37 & $0.7 \%$ & 0.0100 \\
\hline Arthralgia & 32 & $4.3 \%$ & 105 & $2.0 \%$ & 0.0001 \\
\hline Bradycardia & 15 & $2.0 \%$ & 50 & $1.0 \%$ & 0.0087 \\
\hline Chest pain & 97 & $13.1 \%$ & 428 & $8.2 \%$ & $<0.0001$ \\
\hline Colitis & 12 & $1.6 \%$ & 38 & $0.7 \%$ & 0.0126 \\
\hline Diarrhea & 41 & $5.5 \%$ & 186 & $3.6 \%$ & 0.0085 \\
\hline Dyspnea & 43 & $5.8 \%$ & 113 & $2.2 \%$ & $<0.0001$ \\
\hline Edema & 33 & $4.4 \%$ & 157 & $3.0 \%$ & 0.0351 \\
\hline Fatigue, asthenia & 153 & $20.6 \%$ & 746 & $14.2 \%$ & $<0.0001$ \\
\hline Hepatitis & 2 & $0.3 \%$ & 2 & $0.0 \%$ & 0.0225 \\
\hline Interstitial lung disease & 62 & $8.4 \%$ & 87 & $1.7 \%$ & $<0.0001$ \\
\hline Leukopenia & 20 & $2.7 \%$ & 75 & $1.4 \%$ & 0.0100 \\
\hline Neutropenia & 153 & $20.6 \%$ & 837 & $16.0 \%$ & 0.0015 \\
\hline Osteoarthritis & 20 & $2.7 \%$ & 55 & $1.1 \%$ & 0.0002 \\
\hline Pain & 55 & $7.4 \%$ & 227 & $4.3 \%$ & 0.0002 \\
\hline Rash and Acne & 18 & $2.4 \%$ & 72 & $1.4 \%$ & 0.0278 \\
\hline
\end{tabular}


Table 1. Demographic and clinical characteristics (cont.).

\begin{tabular}{|c|c|c|c|c|c|}
\hline \multirow[b]{2}{*}{ Thrombocytopenia } & \multicolumn{2}{|c|}{ Pneumonitis $n=742$} & \multicolumn{2}{|c|}{ No pneumonitis $n=5237$} & \multirow{2}{*}{$\begin{array}{l}p \text {-value } \\
0.0003\end{array}$} \\
\hline & 64 & $8.6 \%$ & 280 & $5.3 \%$ & \\
\hline Vomiting & 56 & $7.5 \%$ & 579 & $11.1 \%$ & 0.0037 \\
\hline \multicolumn{6}{|c|}{ Months from lung cancer diagnosis to CRT initiation } \\
\hline Mean (SD) & 1.8 & $(3.8)$ & 2.0 & $(4.1)$ & 0.2134 \\
\hline Median (min, $\max )$ & 1.1 & $(0,46.6)$ & 1.1 & $(0,48.4)$ & \\
\hline \multicolumn{6}{|c|}{ Months of follow-up from diagnosis of lung cancer } \\
\hline mean (SD) & 28.2 & $(13.7)$ & 25.7 & $(12.1)$ & $<0.0001$ \\
\hline median $(\min , \max )$ & 24.3 & $(9.5,63.2)$ & 22.4 & $(6.9,64.9)$ & \\
\hline \multicolumn{6}{|c|}{ Months of follow-up from initiation of CRT } \\
\hline Mean (SD) & 26.4 & $(13.3)$ & 23.7 & $(11.5)$ & $<0.0001$ \\
\hline Median (min, $\max )$ & 22.5 & $(8.8,58.7)$ & 20.4 & $(4.7,58.9)$ & \\
\hline \multicolumn{6}{|l|}{ Type of CRT received (n, \%) } \\
\hline Sequential CRT & 3 & $0.4 \%$ & 41 & $0.8 \%$ & 0.2588 \\
\hline Concurrent CRT & 739 & $99.6 \%$ & 5196 & $99.2 \%$ & 0.2588 \\
\hline $\begin{array}{l}\text { Patients who have more } \\
\text { than one CRT regimen (n, } \\
\%)\end{array}$ & 19 & 2.6 & 366 & 7.0 & $<0.0001$ \\
\hline $\begin{array}{l}\text { Patients who restarted } \\
\text { CRT after pneumonitis ( } \mathrm{n} \text {, } \\
\% \text { ) }\end{array}$ & 18 & 2.4 & - & - & $\mathrm{N} / \mathrm{A}$ \\
\hline $\begin{array}{l}\text { Type of chemotherapy } \\
\text { used in CRT }(n, \%)\end{array}$ & & & & & 0.0021 \\
\hline Carboplatin + etoposide & 74 & $10.0 \%$ & 478 & $9.1 \%$ & \\
\hline Carboplatin + paclitaxel & 455 & $61.3 \%$ & 3172 & $60.6 \%$ & \\
\hline $\begin{array}{l}\text { Carboplatin + peme- } \\
\text { trexed } \\
\text { disodium }\end{array}$ & 25 & $3.4 \%$ & 388 & $7.4 \%$ & \\
\hline Cisplatin + etoposide & 171 & $23.0 \%$ & 1070 & $20.4 \%$ & \\
\hline $\begin{array}{l}\text { Cisplatin + pemetrexed } \\
\text { disodium }\end{array}$ & 16 & $2.2 \%$ & 126 & $2.4 \%$ & \\
\hline $\begin{array}{l}\text { Cisplatin + vinblastine } \\
\text { sulfate }\end{array}$ & 1 & $0.1 \%$ & 3 & $0.1 \%$ & \\
\hline
\end{tabular}

of follow-up was significantly different between the two groups, the mean follow-up was approximately 2 years post CRT-initiation for both groups indicating sufficient time in both cohorts to have observed a pneumonitis event.

\section{Chemotherapy regimen}

No difference in the duration of chemotherapy prescribed with CRT was observed between patients with or without pneumonitis (median of 42.0 days for both cohorts) nor was there a difference in the number of chemotherapy cycles (median of 7.0 for both cohorts) or the duration of radiation therapy (median of 57.0 vs 56.0 days). Among all patients, median duration of CRT therapy ranged from 28 to 65 days across different first-line regimens, with carboplatin + etoposide having the longest duration.

A multivariate logistic regression analysis showed that the use of carboplatin + pemetrexed, comorbidity index score and the specific baseline comorbidities dyspnea and interstitial lung disease were strong predictors of pneumonitis after CRT treatment $(\mathrm{p}<0.0001)$. The odds of incident pneumonitis with sequential vs concurrent chemotherapy was 0.574 based on a multivariate logistic regression model; however, this finding was not statistically significant $(\mathrm{p}=0.366)$. A summary of these results is shown in Table 2.

Pneumonitis medical management

The mean number of medical management interventions during the follow up period was similar among patients regardless of the type of pneumonitis management approach that was employed (ER admission, inpatient stay or IV steroids). There was, however, a higher frequency of inpatient stays as a medical management approach 


\begin{tabular}{|c|c|c|c|c|}
\hline \multirow[t]{2}{*}{ Predictors of time to incident pneumonitis } & \multicolumn{4}{|c|}{ Total $n=5979$} \\
\hline & $\begin{array}{l}\text { Odds of pneumonitis vs no } \\
\text { pneumonitis }\end{array}$ & Lower CL & Upper CL & p-Value \\
\hline Age at initiation of CRT (years, continuous) & 1.004 & 0.994 & 1.015 & 0.415 \\
\hline Gender (ref: Male) & 0.930 & 0.794 & 1.091 & 0.373 \\
\hline Comorbidity index score at initiation of CRT & 1.061 & 1.037 & 1.085 & $<0.0001$ \\
\hline \multicolumn{5}{|c|}{ CRT chemotherapy type (ref: carboplatin + paclitaxel) } \\
\hline Carboplatin + etoposide & 0.913 & 0.691 & 1.206 & 0.522 \\
\hline Carboplatin + pemetrexed disodium & 0.389 & 0.255 & 0.595 & $<0.0001$ \\
\hline Cisplatin + etoposide & 1.087 & 0.888 & 1.331 & 0.420 \\
\hline Cisplatin + pemetrexed disodium & 0.863 & 0.502 & 1.483 & 0.593 \\
\hline \multicolumn{5}{|l|}{ Comorbidities (ref: no symptoms) } \\
\hline Colitis & 2.052 & 1.039 & 4.055 & 0.039 \\
\hline Dyspnea & 2.183 & 1.493 & 3.192 & $<0.0001$ \\
\hline Bradycardia & 1.452 & 0.785 & 2.688 & 0.235 \\
\hline Edema & 1.210 & 0.808 & 1.812 & 0.356 \\
\hline Endocrinopathy & 1.536 & 0.492 & 4.793 & 0.460 \\
\hline Fatigue, asthenia & 1.338 & 1.090 & 1.643 & 0.005 \\
\hline Interstitial lung disease & 4.652 & 3.286 & 6.586 & $<0.0001$ \\
\hline Respiratory infections & 1.791 & 0.580 & 5.534 & 0.311 \\
\hline Thrombocytopenia & 1.382 & 1.021 & 1.869 & 0.036 \\
\hline \multicolumn{5}{|l|}{ Region (ref: Northeast) } \\
\hline Midwest & 0.881 & 0.688 & 1.128 & 0.315 \\
\hline South & 0.816 & 0.644 & 1.032 & 0.090 \\
\hline West & 0.806 & 0.642 & 1.011 & 0.062 \\
\hline \multicolumn{5}{|c|}{ Type of chemotherapy used in CRT treatment (ref: Concurrent) } \\
\hline Sequential CRT vs Concurrent CRT & 0.574 & 0.172 & 1.913 & 0.366 \\
\hline
\end{tabular}

\section{Table 3. Patients with medical management of pneumonitis.}

\begin{tabular}{|lcc|}
\hline Number of patients with pneumonitis $(\mathrm{n}, \%)$ & 742 & Total $\mathrm{n}=5979$ \\
\hline Medical Management Approaches $(\mathrm{n}, \%)$ & & $12.4 \%$ \\
\hline Emergency room (ER) & 166 & $22.4 \%$ \\
\hline Inpatient & 129 & $17.4 \%$ \\
\hline Intravenous steroids (IV steroids) & 134 & $18.1 \%$ \\
\hline
\end{tabular}

(ER: 1.62, inpatient: 2.12, IV steroids: 1.58). Admission to the ER and inpatient stay was only counted as medical management if an administrative claim for these admissions had codes for pneumonitis on the claim. The proportion of Stage III NSCLC patients with pneumonitis medical management were as follows: ER visits (22.4\%), inpatient hospitalization $(17.4 \%)$ and IV steroids $(18.1 \%)$. Oral steroids were prescribed to $82.7 \%$ of patients with pneumonitis; however, these drugs are widely used for various aspects of cancer management, with $81.2 \%$ of patients without pneumonitis also having a script for oral steroids. A summary of the patients who received medical management of their pneumonitis is shown in Table 3.

\section{All-cause HRU}

Patients with pneumonitis were more frequently admitted to the hospital (33.8 vs 19.2\%, p < 0.0001) and seen in the ER ( 51.9 vs $35.8 \%$, p < 0.0001) for any reason (Table 4). Outpatient visits followed the same trend with significant increases among the patients with pneumonitis versus those without pneumonitis (outpatient visits: 100.0 vs $99.1 \%, \mathrm{p}=0.0088$ ). Office visits were also higher among pneumonitis patients, although the difference was not statistically significant $(92.7$ vs $90.9 \%)$. The number of patients with at least 1 pharmacy claim were 
Table 4. All-cause healthcare resource utilization during the full follow-up period.

\begin{tabular}{|c|c|c|c|c|c|}
\hline & \multicolumn{2}{|c|}{ Pneumonitis $n=742$} & \multicolumn{2}{|c|}{ No pneumonitis $n=5237$} & \multirow[t]{2}{*}{ p-value } \\
\hline \multicolumn{5}{|l|}{ Inpatient PPPM } & \\
\hline Patients with $\geq 1$ inpatient hospitalization & $n=251$ & $33.8 \%$ & $\mathrm{n}=1005$ & $19.2 \%$ & $<0.0001$ \\
\hline $\begin{array}{l}\text { Mean number of inpatient hospitalizations for patients with } \geq 1 \\
\text { inpatient hospitalization (SD, median) }\end{array}$ & 0.170 & $(0.152,0.083)$ & 0.138 & $(0.108,0.083)$ & 0.0023 \\
\hline Mean LOS per hospitalization (SD, median) & 0.416 & $(1.236,0.250)$ & 0.565 & $(2.147,0.167)$ & 0.1493 \\
\hline \multicolumn{6}{|l|}{ ER visits PPPM } \\
\hline Patients with $\geq 1$ ER visit & $\mathrm{n}=385$ & $51.9 \%$ & $\mathrm{n}=1876$ & $35.8 \%$ & $<0.0001$ \\
\hline Mean number of ER visits (SD, median) & 0.207 & $(0.183,0.167)$ & 0.170 & $(0.207,0.083)$ & 0.0005 \\
\hline \multicolumn{6}{|l|}{ Office visits PPPM } \\
\hline Patients with $\geq 1$ clinic/office visit & $\mathrm{n}=688$ & $92.7 \%$ & $n=4762$ & $90.9 \%$ & 0.1076 \\
\hline Mean number of clinic/office visits (SD, median) & 1.182 & $(0.722,1.083)$ & 0.974 & $(0.645,0.833)$ & $<0.0001$ \\
\hline \multicolumn{6}{|l|}{ Outpatients PPPM } \\
\hline Patients with $\geq 1$ outpatients & $\mathrm{n}=742$ & $100.0 \%$ & $n=5189$ & $99.1 \%$ & 0.0088 \\
\hline Mean number of outpatient visits (SD, median) & 3.033 & $(1.649,2.667)$ & 2.507 & $(1.750,2.167)$ & $<0.0001$ \\
\hline \multicolumn{6}{|l|}{ Pharmacy claims PPPM } \\
\hline Patients with $\geq 1$ Rx claim & $n=742$ & $100.0 \%$ & $n=5213$ & $99.5 \%$ & 1.000 \\
\hline Mean Rx claims among patients with $\geq 1$ Rx claim (SD, median) & 6.533 & $(3.322,6.083)$ & 5.879 & $(3.388,5.250)$ & $<0.0001$ \\
\hline Patients with $\geq 1$ steroid claim & $\mathrm{n}=614$ & $82.7 \%$ & $\mathrm{n}=4254$ & $81.2 \%$ & 0.3193 \\
\hline Patients with $\geq 1$ dexamethasone or Solu-Medrol claim & $\mathrm{n}=611$ & $82.3 \%$ & $n=4247$ & $81.1 \%$ & 0.4146 \\
\hline $\begin{array}{l}\text { Patients with } \geq 1 \text { steroid claim } 6 \text { months after the start of CRT } \\
\text { treatment }\end{array}$ & $\mathrm{n}=161$ & $21.7 \%$ & $\mathrm{n}=875$ & $16.7 \%$ & 0.0008 \\
\hline
\end{tabular}

CRT: Chemoradiotherapy; ER: Emergency room; LOS: Length of stay; PPPM: per-patient per-month.

similar between patients with and without pneumonitis; however, those with pneumonitis had significantly more pharmacy claims (mean of 6.53 vs 5.88 PPPM; $\mathrm{p}<0.0001$ ). While the use of steroids over the full study period was similar, use of steroids at least 6 months post CRT treatment was significantly greater among patients with pneumonitis (21.7 vs $16.7 \%$; $\mathrm{p}=0.0008)$.

A sensitivity analysis was performed, comparing all cause HRU for incident pneumonitis patients with one claim for pneumonitis (453 patients) versus patients with two or more claims (289 patients). Patients with two or more claims for pneumonitis had a higher mean number of inpatient visits PPPM (0.18 vs 0.16$)$ but fewer outpatient visits (mean of 1.68 vs 1.61 PPPM, respectively) although these differences were not statistically significant. Only the number of office visits was significantly higher for patients with two or more claims versus patients with one claim (1.31 vs 1.10 PPPM, $\mathrm{p}=0.0002)$. The proportion of patients using steroids was also higher in for patients with two or more claims for pneumonitis $(87.2$ vs $79.9 \%$; $\mathrm{p}=0.0104)$.

\section{All-cause costs}

Mean all-cause total healthcare costs during the full follow-up period were significantly higher for patients with pneumonitis versus those without pneumonitis ( $\$ 4251$ vs $\$ 3969$ PPPM; $\mathrm{p}=0.0163)$, as were medical-specific costs ( $\$ 1153$ vs $\$ 1037$ PPPM; $\mathrm{p}=0.0014$ ) (Table 5). When assessing the component costs, the increase in costs were driven by significantly higher outpatient costs and oral pharmacy costs in pneumonitis patients.

Sensitivity analysis comparing all-cause costs for patients with one claim for pneumonitis versus patients two or more claims found no significant differences between these groups for any setting of care (data not shown).

\section{Discussion/conclusion}

In this retrospective observational study, we found that pneumonitis was observed in $12.4 \%$ of Stage III NSCLC patients receiving CRT. The median time to incident pneumonitis was approximately 6 months after initiating CRT. Medical management for pneumonitis was primarily administered through inpatient stay although there was only a slightly higher frequency of this approach compared with ER visits and administration of IV steroids. Further, these approaches are not mutually exclusive with some patients transitioning from an ER to an inpatient stay or receiving IV steroids in either an ER or inpatient setting. HRU was higher among patients developing pneumonitis and translated into a 7.1\% PPPM total cost increase compared with patients without pneumonitis. 


\begin{tabular}{|c|c|c|c|c|c|c|c|}
\hline \multirow[b]{2}{*}{ Total healthcare costs (mean, SD, median) } & \multicolumn{3}{|c|}{ Pneumonitis $n=742$} & \multicolumn{3}{|c|}{ No pneumonitis $n=5237$} & \multirow{2}{*}{$\begin{array}{l}\text { p-value } \\
0.0163\end{array}$} \\
\hline & $\$ 4251.30$ & $\$ 2974.60$ & $\$ 3476.50$ & $\$ 3969.70$ & $\$ 3044.40$ & $\$ 3220.70$ & \\
\hline Medical costs only & $\$ 1153.20$ & $\$ 899.70$ & $\$ 903.10$ & $\$ 1037.00$ & $\$ 1092.70$ & $\$ 780.00$ & 0.0014 \\
\hline \multicolumn{8}{|l|}{ Component costs (mean, SD, median) } \\
\hline Inpatient & $\$ 298.40$ & $\$ 594.90$ & $\$ 76.00$ & $\$ 291.00$ & $\$ 1348.60$ & $\$ 54.70$ & 0.8970 \\
\hline Emergency room (ER) & $\$ 92.60$ & $\$ 119.80$ & $\$ 54.00$ & $\$ 67.40$ & $\$ 76.40$ & $\$ 44.20$ & 0.0001 \\
\hline Office visit & $\$ 290.80$ & $\$ 273.90$ & $\$ 231.40$ & $\$ 275.30$ & $\$ 303.60$ & $\$ 193.90$ & 0.1716 \\
\hline Outpatient services & $\$ 734.90$ & $\$ 604.10$ & $\$ 565.30$ & $\$ 707.80$ & $\$ 747.10$ & $\$ 487.20$ & 0.2680 \\
\hline Pharmacy & $\$ 3098.20$ & $\$ 2683.30$ & $\$ 2341.50$ & $\$ 2938.40$ & $\$ 2621.80$ & $\$ 2269.30$ & 0.1283 \\
\hline Oral pharmacy costs & $\$ 2840.80$ & $\$ 2637.30$ & $\$ 2061.30$ & $\$ 2594.80$ & $\$ 2553.60$ & $\$ 1873.20$ & 0.0173 \\
\hline
\end{tabular}

The real-world incidence of treatment-related pneumonitis is in line with previously reported observations [2125]. The incidence of pneumonitis in lung cancer patients is reportedly as high as $43 \%$ detected by radiographic means [21], while symptomatic pneumonitis has been found in the range of $5-15 \%$ of patients $[21,22,24,25]$ and approached 30\% in a patient-level meta-analysis [12]. In Radiation Therapy Oncology Group (RTOG) 0617, which found no benefit for high-dose versus standard-dose radiotherapy as a component of concurrent CRT in Stage III NSCLC, incidence of acute and late pneumonitis in the standard-dose CRT (no cetuximab) arm were 9.9 and $13.7 \%$, respectively, for all grades and 4.6 and 1.5\%, respectively for grade 3 or higher events [23]. Our study identified four predictors pneumonitis after CRT, namely use of carboplatin + pemetrexed, comorbidity index score and presence of dyspnea and interstitial lung disease. Pemetrexed use has been previously established as more toxic [25]. Prior studies have provided insight into other risk factors and predictors for treatment-related pneumonitis in the NSCLC population (e.g., age, dose-volume factors, chemotherapy schedule, use of concurrent vs sequential CRT) [23,26] as well as its possible influence on poor overall survival for NSCLC patients [27].

To our knowledge, this is the first published analysis on pneumonitis in a real-world CRT-treated Stage III NSCLC population. In addition to clinical measures (e.g. incidence of pneumonitis, duration of medical management), we examined HRU and costs within the population. For inpatient, ER and outpatient visits, we observed a significant increase in HRU during the follow-up period. This was reflected in an increase in total healthcare costs as well as total medical costs. Overall, our HRU and cost analyses support that patients who develop pneumonitis incur higher healthcare costs, warranting close monitoring to allow for early identification in the Stage III NSCLC population. Given the median time to pneumonitis of almost 6 months, physicians need to monitor patients both during and months following CRT treatment.

Given the nature of retrospective data, known limitations of selection bias and information bias exist. Analyses based on claims data are limited by the lack of clinical details and the use of data that were primarily collected for billing purposes. Enrollment data are not available within the IDV dataset; therefore, continuous enrollment was determined based on claims activity by quarter based on the assumption that patients who had a claim in a given quarter were likely had active enrollment during that quarter. The limitation of this method is that some events may have been missed if there was a change in the patients point of care location or in their benefits enrollment during a quarter where they had claims activity. There are no specific diagnosis codes for NSCLC or lung cancer staging. Stage III NSCLC patients were defined as patients with a lung cancer diagnosis who were treated with drug regimens common for NSCLC and no history of surgical resection or secondary malignancies. Pneumonitis is often a diagnosis of exclusion and may overlap with other pulmonary conditions. To account for that, we used diagnostic codes for pneumonitis as well as pneumonia and other related pulmonary adverse event. Also, we excluded patients who had an event within the first 30 days after index to avoid capturing postobstructive pneumonia which may have led to an underestimation of pneumonitis. Finally, there are significant differences between the design of prospective clinical trials and this retrospective study that may have enriched the population for having these events and therefore make comparison a challenge. Further while this methodology is robust, it also does not account for a full patient history or provider notes that may be more conclusive for pneumonitis but are not available in administrative claims.

Our study documented real-world rates of incident pneumonitis that were in line with rates observed in clinical trials. Patients with pneumonitis had significant increase in HRU and total healthcare costs. While the cost differences observed in this study are statistically significant, the mean cost difference ( $\$ 282$ PPPM) was $<10 \%$ 
and may not be clinically or economically meaningful. The median time to incident pneumonitis was 6 months although pneumonitis could occur within one month or 12 months or more after CRT. The clinical benefit of CRT, a component of the standard of care for Stage III NSCLC that confers improved overall survival in this population, comes with the potential for complications such as pneumonitis, which should be monitored well after the end of the CRT treatment regimen.

\section{Future perspective}

CRT is currently the standard of care for Stage III NSCLC and is likely to continue to be utilized for treatment for the foreseeable future. Using data from real world evidence studies (such as the current study) the potential for complications can be better controlled for through monitoring and early intervention after a patient is treated.

\section{Summary points}

- Patients with Stage III non-small-cell lung cancer (Stage III NSCLC) who receive chemoradiotherapy (CRT) are known to be at risk of radiation-induced pneumonitis, for which the real-world incidence, timing and costs remain unclear.

- In our retrospective claims-based analysis of Stage III NSCLC patients treated with CRT, treatment-related pneumonitis developed in $12.4 \%$ of patients after a mean of 177 days (6 months).

- Duration of chemotherapy, number of chemotherapy cycles and duration of radiation therapy did not differ between patients with versus without pneumonitis.

- Conversely, use of carboplatin + pemetrexed, comorbidity index score and baseline dyspnea and interstitial lung disease were strong predictors of pneumonitis after CRT $(p<0.0001)$.

- Patients with versus without pneumonitis were more frequently admitted to the hospital (33.8 vs $19.2 \%$, $\mathrm{p}<0.0001)$ and seen in the emergency room (51.9 vs $35.8 \%, \mathrm{p}<0.0001)$.

- Additionally, patients with pneumonitis had significantly more pharmacy claims (mean of 6.53 vs 5.88 PPPM; $\mathrm{p}<0.0001)$ and use of steroids at least 6 months post CRT treatment $(21.7$ vs $16.7 \% ; p=0.0008)$.

- From a cost standpoint, pneumonitis was associated with significant increases in mean all-cause total healthcare costs during the full follow-up period ( $\$ 4251$ vs $\$ 3969$ PPPM; $p=0.0163$ ) and medical-specific costs $(\$ 1153$ vs $\$ 1037$ PPPM; $p=0.0014)$.

- These real-world results are consistent with incidence rates derived from clinical trials and support monitoring for pneumonitis beyond completion of CRT.

\section{Author contributions}

KJ Ryan and B Seal were responsible for study conception, design, data interpretation and drafting, and revision of the manuscript. D Nero, CH Lee and JK Kish were responsible for the study design, data manipulation, analytic reporting and drafting of the manuscript. BA Feinberg, R Pimentel and A Gajra provided clinical guidance and provided support in the revision of the manuscript.

Financial \& competing interests disclosure

KJ Ryan and B Seal are employed by AstraZeneca and owns stock in AstraZeneca. AstraZeneca sponsored the study and provided financial support for the conduct of the research and for preparation of the article. The authors have no other relevant affiliations or financial involvement with any organization or entity with a financial interest in or financial conflict with the subject matter or materials discussed in the manuscript apart from those disclosed.

Writing assistance was provided by L Orloski, PharmD (independent medical writer), which was funded by Cardinal Health.

\section{Open access}

This work is licensed under the Attribution-NonCommercial-NoDerivatives 4.0 Unported License. To view a copy of this license, visit http://creativecommons.org/licenses/by-nc-nd/4.0/

\section{References}

Papers of special note have been highlighted as: $\bullet$ of interest; $\bullet \bullet$ of considerable interest

1. National Comprehensive Cancer Network. NCCN Clinical Practice Guidelines in Oncology (NCCN Guidelines $\left.{ }^{\circledR}\right)(2019)$. www.nccn.org/professionals/physician_gls/pdf/nscl.pdf.

2. SEER Cancer Statistics Review 1975-2016.

https://seer.cancer.gov/csr/1975_2016/browse_csr.php?sectionSEL=15\&pageSEL=sect_15_table.08. 
3. Byhardt RW, Scott CB, Ettinger DS et al. Concurrent hyperfractionated irradiation and chemotherapy for unresectable nonsmall cell lung cancer. Results of Radiation Therapy Oncology Group 90-15. Cancer 75(9), 2337-2344 (1995).

4. Dillman RO, Seagren SL, Propert KJ et al. A randomized trial of induction chemotherapy plus high-dose radiation versus radiation alone in stage III nonsmall cell lung cancer. N. Engl. J. Med. 323, 940-945 (1990).

5. Lee JS, Scott C, Komaki R et al. Concurrent chemoradiation therapy with oral VP-16 and cisplatin for locally advanced inoperable nonsmall cell lung cancer: RTOG Protocol 91-06. J. Clin. Oncol. 14(4), 1055-1064 (1996).

6. Dhokarh R, Li G, Schmickl CN et al. Drug-associated acute lung injury: a population-based cohort study. Chest 142(4), 845-850 (2012).

7. Sakurada T, Kakiuchi $S$, Tajima $S$ et al. Characteristics of and risk factors for interstitial lung disease induced by chemotherapy for lung cancer. Ann. Pharmacother. 49(4), 398-404 (2015).

8. Kim JY, Kim YS, Kim YK et al. The TGF-beta1 dynamics during radiation therapy and its correlation to symptomatic radiation pneumonitis in lung cancer patients. Radiat. Oncol. 4, 59 (2009).

9. Niu X, Li H, Chen Z et al. A study of ethnic differences in TGFbetal gene polymorphisms and effects on the risk of radiation pneumonitis in non-small-cell lung cancer. J. Thorac. Oncol. 7(11), 1668-1675 (2012).

10. Rodrigues G, Lock M, D'Souza D, Yu E, Van Dyk J. Prediction of radiation pneumonitis by dose volume histogram parameters in lung cancer: a systematic review. Radiother. Oncol. 71(2), 127-138 (2004).

11. Antonia SJ, Villegas A, Daniel D et al. Durvalumab after chemoradiotherapy in stage III non-small-cell lung cancer. $N$ Engl J Med. 377(20), 1919-1929, (2017).

- Randomized Phase III trial for durvalumab, a PD-L1 inhibitor that has become the new standard of care as consolidation therapy after chemoradiation for unresectable stage III non-small-cell lung cancer (NSCLC).

12. Palma DA, Senan S, Tsujino K et al. Predicting radiation pneumonitis after chemoradiation therapy for lung cancer: an international individual patient data meta-analysis. Int. J. Radiat. Oncol. Biol. Phys. 85(2), 444-450 (2013).

- Systematic review of treatment-related risk factors for symptomatic radiation pneumonitis after chemoradiation for lung cancer.

13. Symphony Integrated Dataverse. https://symphonyhealth.com/wp-content/uploads/2015/10/IDV-Product-Introduction.pdf.

14. Larici AR, del Ciello A, Maggi F et al. Lung abnormalities at multimodality imaging after radiation therapy for non-small cell lung cancer. Radiographics 31(3), 771-789 (2011).

15. Williams JP, Johnston CJ, Finkelstein JN. Treatment for radiation-induced pulmonary late effects: spoiled for choice or looking in the wrong direction? Curr. Drug Targets 11(11), 1386-1394 (2010).

16. CMS ASP Drug Pricing.

www.cms.gov/Medicare/Medicare-Fee-for-Service-Part-B-Drugs/McrPartBDrugAvgSalesPrice/2017ASPFiles.html.

17. First Databank. www.fdbhealth.com/policies/drug-pricing-policy/.

18. CMS MPFS Booklet. www.cms.gov/Outreach-and-Education/Medicare-Learning-Network-MLN/MLNProducts/downloads/How_t o_MPFS_Booklet_ICN901344.pdf.

19. US Department of Health and Human Services, Centers for Medicare and Medicaid Services: Hospital Outpatient PPS. Addendum A and Addendum B Updates. www.cms.gov/HospitalOutpatientPPS/AU/list.asp.

20. HCUP Cost to Charge. www.hcup-us.ahrq.gov/db/state/costtocharge.jsp.

21. Abid SH, Malhotra V, Perry MC. Radiation-induced and chemotherapy-induced pulmonary injury. Curr. Opin. Oncol. 13(4), 242-248 (2001).

22. Antonia S, Goldberg SB, Balmanoukian A et al. Safety and antitumour activity of durvalumab plus tremelimumab in non-small cell lung cancer: a multicentre, Phase 1b study. Lancet Oncol. 17(3), 299-308 (2016).

23. Bradley JD, Paulus R, Komaki R et al. Standard-dose versus high-dose conformal radiotherapy with concurrent and consolidation carboplatin plus paclitaxel with or without cetuximab for patients with stage IIIA or IIIB non-small-cell lung cancer (RTOG 0617): a randomised, two-by-two factorial Phase 3 study. Lancet Oncol. 16(2), 187-199 (2015).

-. Randomized Phase III trial, providing insight into the incidences of acute and late pneumonitis with high-dose versus standard-dose radiotherapy as a component of concurrent chemoradiation for stage III NSCLC.

24. Curran WJ Jr, Paulus R, Langer CJ et al. Sequential vs. concurrent chemoradiation for stage III non-small cell lung cancer: randomized Phase III trial RTOG 9410. J, Natl Cancer Inst. 103(19), 1452-1460 (2011).

-• Randomized Phase III trial, providing insight into acute and late pulmonary toxicity with sequential versus concurrent chemoradiation for stage III NSCLC.

25. Kong FM, Ten Haken R, Eisbruch A, Lawrence TS. Non-small cell lung cancer therapy-related pulmonary toxicity: an update on radiation pneumonitis and fibrosis. Semin. Oncol. 32(Suppl 3), S42-S54 (2005).

26. Nishino M, Chambers ES, Chong CR et al. Anti-PD-1 inhibitor-related pneumonitis in non-small cell lung cancer. Cancer Immunol. Res. 4(4), 289-293 (2016).

- Case report describing the radiographic workup and clinical course of PD-1 inhibitor-related pneumonitis in advanced NSCLC. 
27. Dang J, Li G, Zang S, Zhang S, Yao L. Risk and predictors for early radiation pneumonitis in patients with stage III non-small cell lung cancer treated with concurrent or sequential chemoradiotherapy. Radiat. Oncol. 9, 172 (2014).

- Observational study focused on identifying risk factors and predictors with concurrent versus sequential chemoradiation for stage III NSCLC. 
